\title{
Morfologia dos vasos da base do encéfalo do quati (Nasua nasua) ${ }^{1}$
}

\author{
Juliana Regina Barreiro², Ana Flavia de Carvalho ${ }^{3}$, André Luis R. Franciolli ${ }^{*}$, Guilherme \\ José B.C. Ferreira ${ }^{5}$, Jussara R. Ferreira ${ }^{6}$, Carlos Eduardo Ambrósio ${ }^{7}$ e Maria A. Miglino ${ }^{4}$
}

\begin{abstract}
Barreiro J.R., Carvalho A.F., Franciolli A.L.R., Ferreira G.J.B.C., Ferreira J.R. Ambrósio C.E. \& Miglino M.A. 2012. [Morphology of the main vessels of the coati brain ( $\mathrm{Na}$ sua nasua).] Morfologia dos vasos da base do encéfalo do quati (Nasua nasua). Pesquisa Veterinária Brasileira 32(6):567-572. Setor de Anatomia dos Animais Domésticos e Silvestres, Departamento de Cirurgia, Faculdade de Medicina Veterinária e Zootecnia, Universidade de São Paulo, Av. Prof. Orlando Marques de Paiva 87, São Paulo, SP 05508-270, Brazil. E-mail: andrefranciolli@usp.br

The morphology of the brain of coati, Nasua nasua, was studied, to compare the findings with other species described. The brains of five coatis were used, proceeding from the Scientific Breeding School (Cecrimpas), Unifeob. The animals were sacrificed in accordance with the legislation (Cobea). With a needle, the common carotid artery and the external jugular vein was cannulated to cranial direction, injected latex solution stained with colored red barium respectively. Afterwards the animals were fixed in $10 \%$ formaldehyde solution. Brain has its dependent nutrition of four arteries, the internal carotid arteries and the right and left vertebral arteries. These vessels had composed the basal and carotid circuits that anastomose through the cerebral arteries volumes. They run in the base of the brain under piamater meninges.
\end{abstract}

Index terms: Nasua nasua, coati, encephalic vascularization.

RESUMO.- Estudou-se a morfologia do encéfalo de Nasua nasua - quati, buscando comparar estes achados com outras espécies já descritos. Foram utilizados cinco encéfalos de quatis, provenientes do Criatório Científico (Cecrimpas), Unifeob. Os animais foram eutanásiados de acordo com a legislação (Cobea). Canulou-se a artéria carótida comum e a veia jugular externa sentido cranial, injetou-se solução de

\footnotetext{
${ }^{1}$ Recebido em 11 de novembro de 2011.

Aceito em 1 de março de 2012.

${ }^{2}$ Departamento de Nutrição e Produção Animal, Faculdade de Medicina Veterinária e Zootecnia (FMVZ), Universidade de São Paulo (USP), Av. Duque de Caxias Norte 225, Pirassununga, SP 13635-900, Brasil.

${ }^{3}$ Centro Universitário da Fundação de Ensino Octavio Bastos, Av. Dr. Octávio Bastos s/n, Jardim Nova São João, São João da Boa Vista, SP 13870000, Brasil

${ }^{4}$ Departamento de Cirurgia, FMVZ-USP, Av. Prof. Dr. Orlando Marques de Paiva 87, Cidade Universitária, São Paulo, SP 05508-270, Brasil. *Autor para correspondência: andrefranciolli@usp.br

${ }^{5}$ Universidade Federal do Piauí, Planalto Horizonte, PI 64900-000, Brasil.

${ }^{6}$ Faculdade de Medicina, Universidade de Brasília, Campus Darcy Ribeiro, Asa Norte, Brasília, DF 70910-000, Brasil.

${ }^{7}$ Departamento de Ciências Básicas, Faculdade de Zootecnia e Engenharia de Alimentos, USP, Av. Duque de Caxias Norte 225, Campus da USP, Pirassununga, SP 13635-900, Brasil.
}

látex/bário corado de vermelho na artéria carótida, e solução de látex corado de azul na veia jugular. Em seguida os animais fixados em solução de formaldeído a $10 \%$. 0 encéfalo tem sua nutrição dependente de quatro artérias, as artérias carótidas internas e as artérias vertebrais direitas e esquerdas. Esses vasos compuseram o circuito basilar e carotídeo que se anastomosam através das artérias cerebrais caudais. Correm na base do encéfalo sob a meninge pia mater.

TERMOS DE INDEXAÇÃO: Nasua nasua, quati, vascularização encefálica.

\section{INTRODUÇÃO}

Nasua nasua conhecido popularmente como quati_assim como o mão-pelada (Procyon cancrivorus) pertence à família dos mascarados, Filo Chordata, Classe Mammalia, Ordem Carnívora e Família Procyonydae. Esta família é composta de 7 gêneros com 19 espécies, ocorrendo em quase todo o mundo. No Brasil são encontrados principalmente em áreas de floresta. São animais onívoros de hábitos diurno que ao entardecer recolhem-se as copas das árvores onde permanecem até o amanhecer (Beisiegel 2001, Franciolli et al. 2007, Bellatine et al. 2010). 
Estes animais podem pesar de 3-11 kg exibindo comprimento corporal de $41-67 \mathrm{~cm}$ e comprimento da cauda de $32-60 \mathrm{~cm}$. 0 pêlo é comprido e áspero, com variados matizes cinzentos, avermelhados e amarelados, com manchas arredondadas em torno dos olhos, e as orelhas apresentam margens amareladas. A cauda tem como características cerca de oito anéis claros, alternados com outros escuros. Os membros pélvicos são mais longos que os torácicos. Vivem cerca de 12 anos e reproduzem-se uma vez ao ano. Há quatro espécies encontradas desde o Panamá (América Central) até a Argentina (Russel 1996, Beisiegel 2001, Gompper 2004, Pieri et al. 2011).

Estudos contemporâneos (Fawcett \& Blanchford 1905/1906, Kramer 1912, McKenna, 1966, Gillilam 1969, Guerin et al. 1976, Bugge 1974, Puchardes-Orts et al. 1975) sustentam que a complexidade morfológica cerebral é mais ou menos evoluída, e isto se reflete no modelo de aporte sanguíneo, visto que há dois fatores importantes a se considerar: a regressão do lobo olfatório e o acréscimo de camadas de neocórtex.

Alguns autores (Ferreira 1998, Reckziegel 2001, Ferreira 2002, Campos et al. 2003, Ferreira \& Prada 2005, Paiva-de-Souza et al. 2010) após estudar em ungulados os arranjos apresentados pelas artérias encefálicas, destacaram que variados grupos de animais têm despertado muito interesse desde épocas remotas. Um modelo de classificação desses arranjos foi proposto por Tandler (1898), demonstrando que a filogenia e a ontogenia dessas artérias modificam-se em paralelismo com a evolução das exigências funcionais do sistema nervoso central. Com base nesta proposta e em relação ao modelo arquitetônico do suprimento sanguíneo do sistema nervoso central dos animais, estudos filo e ontogênicos de De Vriese (1905), Testut (1911) e Bugge (1974) mostraram claramente que, no processo evolutivo, processam-se modificações cuja tendência é responder as exigências, no sentido de que o Sistema Nervoso Central (SNC) possa desempenhar adequadamente o seu papel. A evolução cerebral não teria sido possível se não fosse acompanhada por uma correspondente adaptação dos padrões vasculares, decorrentes de modificações no modo de ramificação das artérias encefálicas que respondem pelas eventuais modificações nos mecanismos neurovasculares conquistados recentemente.

Alcântara (1997) e Lima (2006) estudaram nos mamíferos e carnívoros o circulo de Willis a partir da classificação de Tandler (1898), que agrupou em três tipos fundamentais, os modelos de vascularização do encéfalo e medula espinhal dos vertebrados atuais. No primeiro tipo os vasos dependem quase que totalmente do circuito basilar e mantém em muitos casos uma rede admirável, ou no período embrionário ou no adulto, ao nível da artéria maxilar; no tipo dois, a vascularização é dependente de dois circuitos: o basilar e o carotídeo; e no tipo três as artérias carótidas são praticamente ausentes na irrigação do encéfalo.

0 modelo primata foi estudado no homem por De Vriese (1905) e Testut (1911), entre outros, com base na classificação de Tandler (1898). A partir de então a literatura corrente (Rouviére 1959, Lockhart \& Hamilton 1983, Machado 1988, Rohen \& Yokochi 1993, Casals et al 2011) trata descrição e ilustração dos sistemas carotídeos e vertebro basilar interligados pelas artérias comunicantes caudais e rostrais, e dependente do suprimento de sangue via artérias carótidas internas e vertebrais nos dois antimeros. Evidências indicam que a partir dos prossímios, com base em estudos em Perodicticus potto e Nycticebus tardigradus (Beddard 1904) e em Nycticebus Couang couang (Kramer 1912) alguns animais retiveram um sistema primitivo de circulação a partir de uma ramificação lateral da artéria carótida interna que corre ao longo da porção petrosa da base occipital, que Mckenna (1966) refere-se como o vaso formador das artérias estapedial e promotorial, que foram mudando de configuração para construir o novo modelo primata descrito em chimpanzé (Glidem \& Degaris 1936, Shellshear 1930), no orangotango (Shellshear 1927) e em macaco rhesus (Kassel \& Langfitt 1965), além de outros primatas neotropicais, o sagüi (Miraglia \& Teixeira,1960, Souza et al. 1962), e o macaco prego (Ferreira et al. 2005).

Estudos comparativos em diferentes espécies vêm demonstrando que não apenas o sistema nervoso, como também o arranjo vascular deste, encontram-se em processo contínuo de modificação. Desse modo há necessidade de serem realizadas pesquisas sobre o comportamento do arranjo vascular encefálico, na série animal com fins de fornecer subsídios para o conhecimento das espécies não estudadas, sempre visando inserções diretas e indiretas destas informações nas ciências da vida.

\section{MATERIAL E MÉTODOS}

Foram utilizados cinco encéfalos de quati (Nasua nasua), provenientes do Criatório Científico (Cecrimpas) situado na Fazenda Escola do Centro Universitário da Fundação de Ensino Octavio Bastos (Unifeob), autorizado pelo IBAMA (Proc.02027.002322/98-99) e pelo comitê de ética da instituição. Os animais foram eutanasiados conforme os princípios éticos da utilização de animais para fins de pesquisa, didática e clínica, adaptado pelos termos do Colégio Brasileiro de Experimentação Animal (Cobeas). Todo o procedimento ocorreu no Laboratório de Anatomia e Radiologia do Centro Universitário da Fundação de Ensino Octávio Bastos (Unifeob).

Após a eutanásia, canulou-se a artéria carótida comum e a veia jugular externa, ambas sentido cranial, com auxílio de uma cânula 25x7 e uma seringa de $10 \mathrm{ml}$, nas quais injetou-se solução de látex (Neoprene $囚$ ) corado de vermelho com Bário, na proporção de 1:1 na artéria carótida, e solução de látex azul na veia jugular, em seguida os animais foram radiografados. Após este procedimento, realizou-se a fixação em solução de formaldeído a 10\%.

Após a fixação, os animais tiveram seus encéfalos retirados. A calota craniana foi serrada, mantendo a integridade das meninges e estruturas anexas. Os encéfalos foram dissecados com auxílio de Lupa (Zeiss ${ }^{\circledR}$, modelo Stemi DV4) e fotodocumentados com câmera digital $\left(\right.$ Nikon $\left.^{\circledR}\right)$ para posterior análise e descrição. A terminologia utilizada teve como base a Nomina Anatômica Veterinária (WAVA 1992).

\section{RESULTADOS E DISCUSSÃO}

A análise dos resultados nos permitiu considerar que o encéfalo é suprido por quatro artérias principais: as carótidas internas e as vertebrais direita e esquerda (Fig.1A). Os vasos derivados da subdivisão das artérias carótidas, nos dois 
antímeros, compuseram o circuito arterial rostral da base do encéfalo do quati, também chamado de circuito carotídico em carnívoros (Alcantâra 1992, Casal et al. 2005 Lima 2006) nos ungulados (Ferreira 1998, Reckziegel et al. 2001, Ferreira \& Prada, 2005) e em primatas (Ferreira 1997, Ferreira \& Prada, 2001, Silva \& Ferreira 2002); os vasos derivados das subdivisões das artérias vertebrais compuseram o circuito arterial caudal ou vértebro basilar relativamente parecido ao de outros carnívoros (Alcantâra 1997, Casal et al. 2005, Lima 2006) conforme outros estudos.

0 aporte sangüíneo do encéfalo assemelhou-se ao descrito por Campos et al. (2003) em eqüinos, Ferreira \& Prada (2005) em suínos, Reckziegel et al. (2001) em capivaras, Lima (2006) em gatos, Tandler (1898), Silva \& Ferreira (2002) no macaco prego, sendo este sistema o carótido e o basilar, com exceção Reckziegel et al. (2001) em capivaras, onde citam que a vascularização do encéfalo esta na dependência única do sistema vertebro-basilar. Testut (1911) relata que nas fases iniciais do desenvolvimento filogenético e ontogenético, a artéria carótida interna é a única artéria a conduzir sangue à massa encefálica; a artéria vertebral não existe.

Ao estudarem as artérias da base do encéfalo de primatas (Cebus apella) (Ferreira 1997, Ferreira \& Prada 2001, Ferreira et al. 2005) autores consideraram o sistema vertebral como responsável pela formação da artéria basilar, o mesmo critério utilizado por De Vriese (1905), enquanto Ferreira (1998) estudando as artérias da base do encéfalo de suínos (Sus scrofa domesticus) considerou a artéria vertebral como um vaso suplementar na formação da artéria basilar, que no suíno apresenta-se como um ramo rostral da artéria occipital, dando a está uma maior importância. Em nossos estudos acreditamos que ambas as artérias têm a mesma importância funcional.

Nos animais estudados, as artérias da base do encéfalo mostraram-se dispostas de maneira a caracterizarem uma situação intermediária entre os estágios médio e final citado por Testut (1911). 0 padrão vascular das artérias da base do encéfalo dos quatis estudados tende ao tipo 2 alfa proposto por Tandler (1898) e referido por De Vriese (1905), onde a artéria carótida interna divide-se em um ramo terminal cranial e um caudal, artéria comunicante caudal, mas esta última não se implanta mais na artéria basilar, pois termina na artéria caudal do cérebro e anastomosa-se com o ramo de divisão da artéria basilar, resultante da união das artérias vertebrais. Nestes casos as artérias cerebrais rostral, média e caudal provêm, ainda, das artérias carótidas internas. Este tipo é encontrado em alguns carnívoros (De Vriese 1905), o qual considerou como pertencentes aos Tipos 2 e 3 , e estes encontram-se em posições extremas na evolução filogenética. Neste grupo encontram-se aqueles em que todo o suprimento sanguíneo arterial para o encéfalo provém das artérias carótidas internas, aqueles em que este suprimento é realizado pelas artérias vertebrais e uma série de tipos de transição entre estes dois grupos, a ponto de não se poder estabelecer nenhuma escala evolutiva, pois uma mesma disposição existe nos grupos filogenéticos mais afastados e, ao contrário, numa mesma ordem encontram-se disposições do círculo arterial que vão de um extremo a outro.
A artéria basilar bifurca-se na artéria cerebral posterior, por nos classificada como artéria rostral do cérebro, assim como nos eqüinos da raça Puro Sangue Inglês e no Gallus gallus descrito por Silva \& Ferreira (2002), e em macacos por De Vriese (1905) e Casals et al. (2011) e no Meleagris gallopavo (Carvalho \& Campos 2011), ligadas às carótidas internas pelas comumente caudal, e estas seguem dorsolateralmente a artéria basilar e no sentido dorsal á medula oblonga até artéria vertebral. Após a formação da artéria basilar surge à artéria caudal do cerebelo; esta segue dorsolateralmente passando pelas raízes do nervo hipoglosso situado dorsalmente à medula oblonga. A artéria média do cerebelo é emitida lateralmente da artéria basilar antes desta atingir o corpo trapezóide, após sua origem a mesma segue lateralmente em relação ao nervo abducente, que está posicionado ventralmente (Fig.1A).

De acordo com Ferreira (1997) a artéria cerebral posterior corresponde ao ramo caudal da artéria carótida interna, nos peixes, anfíbios, répteis e aves, aparecendo nos mamíferos primitivos como uma ramificação colateral da parte caudal da artéria carótida, no entanto, para Testut (1911), em primatas, a artéria cerebral posterior é o ramo de bifurcação final da artéria basilar, primariamente considerada como pertencente ao sistema carótido e na evolução passa para o domínio da artéria vertebral.

A artéria carótida interna origina a artéria cerebral rostral formando o quadrame rostrolateral do Círculo arterial do cérebro ou Círculo de Willis, semelhante em gatos como descrito por Lima (2006), o qual continua se encurvando sobre a face medial do hemisfério (Fig.3A). Por sua vez o Círculo de Willis, que circunda a hipófise e o quiasma óptico, é formado, além da artéria cerebral, pelas artérias comumente rostral e caudal (Fig.2B). Este sistema foi considerado por Testut (1911) como heptágono de Willis, pois o conjunto de vasos anastomosados não é identificado morfologicamente como um círculo, mas como um heptágomo. Podemos dizer que estas anastomoses são estáveis, ou seja, se apresentaram presentes sem anomalias, relacionados ao ponto de vista ontogenético e filogeneticamente.

A artéria oftálmica interna deixa a artéria carótida interna como um vaso muito delicado, como descrito em capivaras por Reckziegel et al. (2001), este vaso transcorre na superfície ventral do quiasma óptico e volta para a superfície dorsal do nervo óptico (Fig.1A,C, Fig.2A). A artéria oftálmica é descrita por Ferreira (1997) como um ramo da artéria cerebral anterior, descrita por nos como artéria cerebral rostral. De Vriese (1905), Gillilan (1969) consideraram que nesse seguimento as variáveis estão na dependência do estágio evolutivo do sentido do olfato.

Ferreira \& Prada (2005) observaram varias arteríola no encéfalo de suínos o mesmo observado no quati por nos classificado como ramos corticais, os quais irrigam todo o córtex cerebral e ramos que se direcionam para a ponte (Fig.2A,B).

A artéria rostral do cerebelo, citada como artéria cerebelar rostral por Carvalho (2006), deixa a artéria comumente caudal próximo à sua terminação e união com a artéria basilar como descrito por Nanda (1975) e corre 

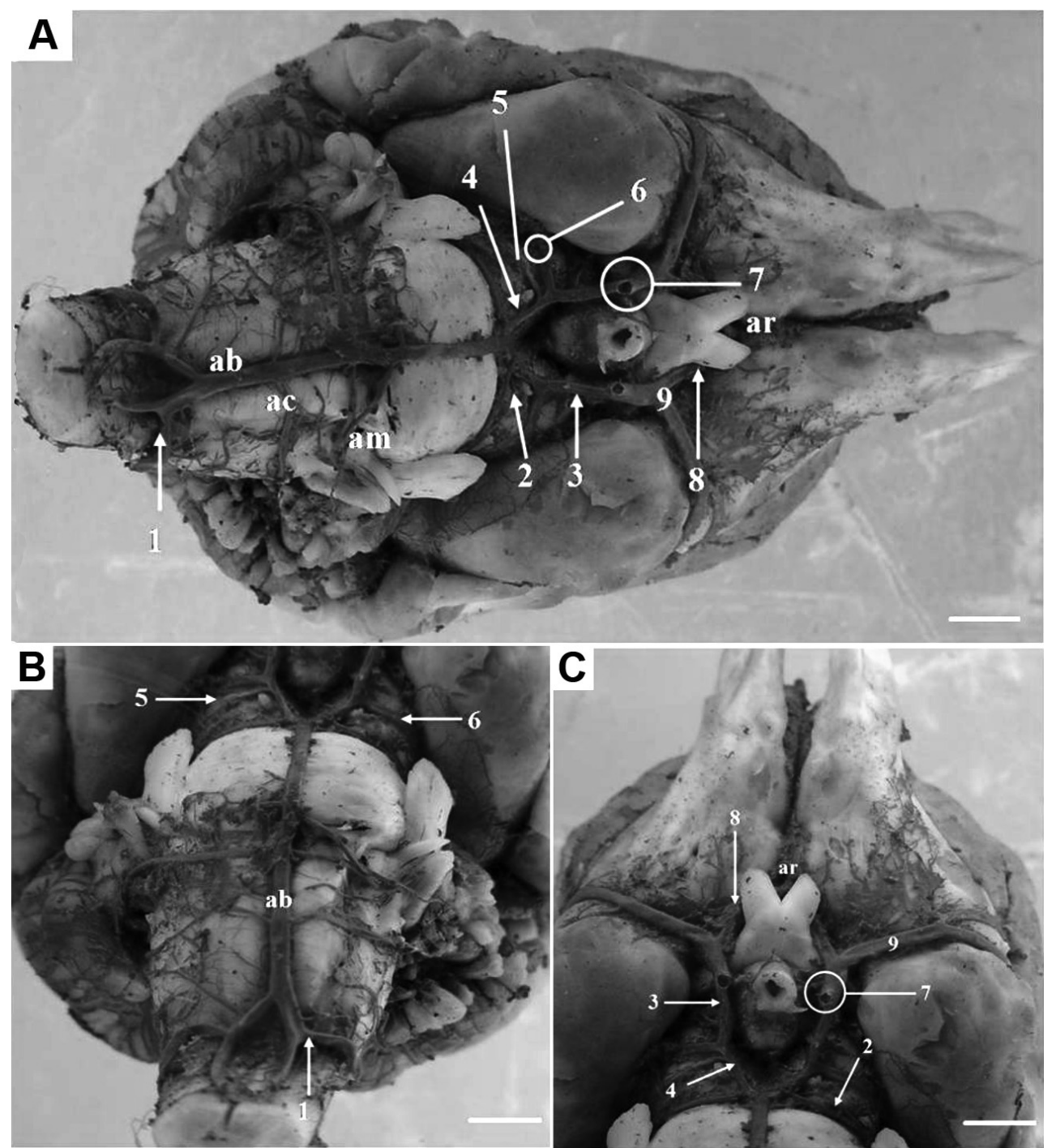

Fig.1. (A-C) Artérias da base do encéfalo do quati. Artéria basilar (ab), Artéria carótida interna (7), Artéria comumente caudal (3), Artéria caudal do cérebro (6), Artéria rostral do cerebelo (5), Artéria mesencefálica (4), Artéria rostral do cérebro (2), Artéria média do cerebelo (am), Artéria caudal do cerebelo (ac), Artéria média do cérebro (9), Artéria oftálmica interna (8), Artéria cerebral rostral (ar), Artéria vertebral (1). Barras: $1 \mathrm{~cm}$.
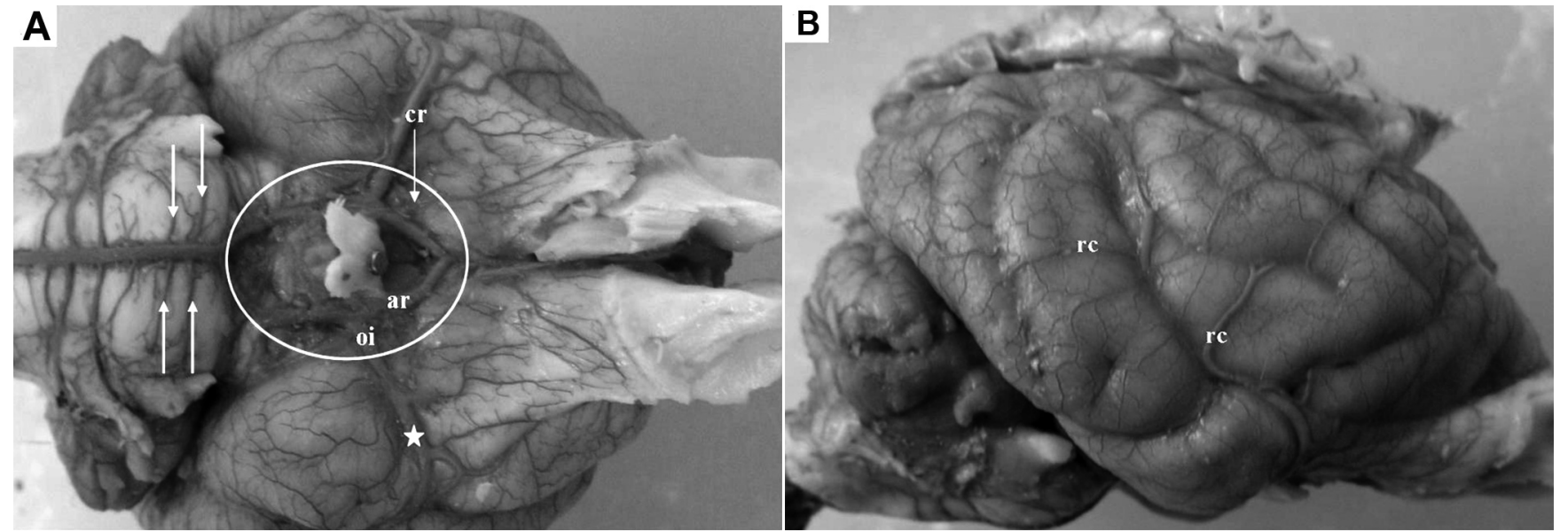

Fig.2. (A,B) Artérias encéfalo quati. Arterial cerebral rostral (ar), Artéria comunente rostral (cr), Artéria media do cérebro (estrela), Artéria oftálmica interna (oi), Ramos para a ponte (seta), Ramos corticais (rc), Círculo arterial do cérebro ou Círculo de Willis (círculo). Barras: $1 \mathrm{~cm}$. 


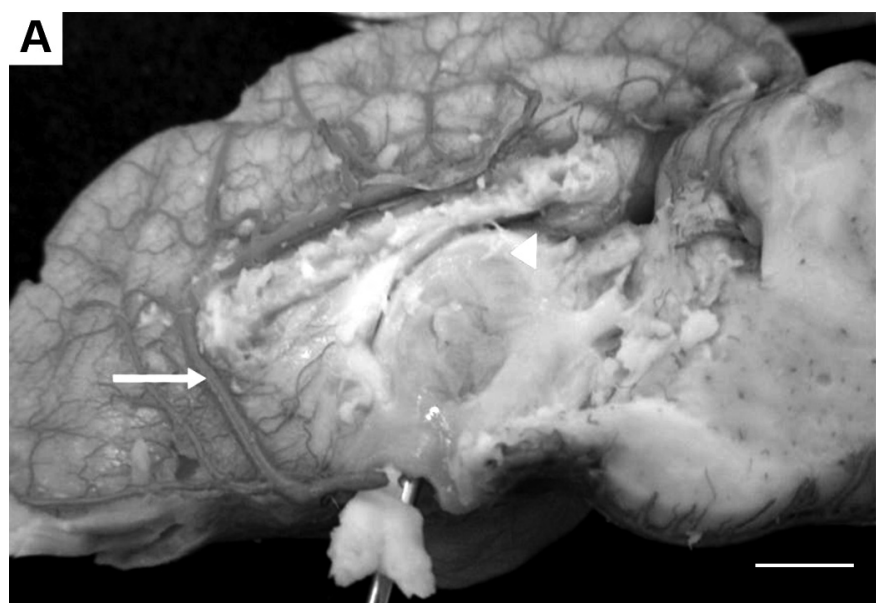

Fig.3. Artérias do encéfalo (hemisfério esquerdo removido). Artéria cerebral rostral (seta), Artéria caudal do cérebro (cabeça de seta). Barra: $1 \mathrm{~cm}$.

sentido dorsolateralmente, e a mesma percorre o pilar do encéfalo até alcança o espaço entre o cerebelo. Já a artéria comunicante caudal é o ramo caudal da artéria carótida interna referida por De Vriese (1905) como artéria comunicante posterior representando morfologicamente os ramos caudais da artéria carótida interna ou cerebrais primitivas e aparece na filogenia à medida que o sistema nervoso evolui, o segmento distal da artéria comunicante caudal pode ser considerada como artéria mesencefálica de acordo com Nanda (1975). A artéria caudal do cérebro curva-se dorsolateralmente para ascender ao longo do encéfalo, chegando sob a parte caudal do lobo piriforme, semelhante ao encontrado por Casal et al. (2005) em Canis familiaris e considerada por Nanda (1975) como um dos ramos da artéria comumente caudal (Fig.1, Fig.3A).

Em conclusão, do estudo sobre e vascularização do encéfalo de quati, julgamos que o sistema de aporte sangüíneo assemelhou-se ao já descrito na literatura, sendo este sistema o carótido e o basilar. As artérias da base do encéfalo mostrarem-se dispostas a caracterizar uma situação intermediária entre os estágios médio e final de vascularização, e que o padrão vascular das artérias da base do encéfalo dos quatis estudados tende ao tipo 2 alfa. 0 encéfalo e os arranjos dos vasos através da literatura consultada encontram-se em contínuo processo de modificação, e que o comportamento das artérias encefálicas, comparativamente entre as espécies, exibe um modelo básico, ao qual são acrescentadas modificações relativas aos diferentes grupos de animais.

\section{REFERÊNCIAS}

Alcântara M.A. 1997. Estudo anatômico da artéria cerebral rostral em cães (Canis familiares, Linnaeus, 1758): origem, trajeto, ramificação e distribuição. Tese de Doutorado, Faculdade de Medicina Veterinária e Zootecnia, USP, São Paulo. 151p.

Alcântara M.A. 1992. Estudo anatômico das artérias da base do encéfalo de cães (Canis familiares Linnaeus, 1758). Tese de Mestrado, Faculdade de Medicina Veterinária e Zootecnia, USP, São Paulo. 101p.

Beddard F.E. 1904. Note on the brains of the Potto (Perodicticus potto) and the Slow loris (Nycticebus tardigradus) with some observations upon the arteries of the brain in certain primates. Proc. Zoological Society of London 1:157-63.
Beisiegel B.M. 2001. Notes on the coati, Nasua nasua (Carnívora: Procyonidae) in Atlantic Forest area. Braz. J. Biol. 61(4):689-692.

Bellatine T., Mançanares C.A.F., Franciolli A.L.R., Ambrósio C.E., Martins D.S., Miglino M.A. \& Carvalho A.F. 2010. Estudo morfofuncional das glândulas mamárias de Mão Pelada, Procyon cancrivorus. Pesq. Vet. Bras. 30(8):689-695.

Bugge J. 1974. The cephalic arterial system in insectivores, primates, rodents and lagomorphs, with special reference to the systematic classification. Acta Anat. 87:1-160.

Campos A., Prada I.L.S., Junior I.S. \& Santos D. 2003. Artérias da base do encéfalo de eqüinos. Sistema occipito-basilar. Braz. J. Vet. Res. Anim. Sci. 40:107-117.

Carvalho S.M.F. 2006. Contribuição da imagem funcional por ressonância magnética para o estudo da reorganização do córtex motor pós-AVCI. Dissertação de Mestrado, Faculdade de Medicina, Ribeirão Preto, SP. 116p.

Carvalho A.D. \& Campos R. 2011. A systematic study of the brain base arteries in the turkey (Meleagris gallopavo). Pesq. Vet. Bras. 31(Supl.1): 39-46.

Casal D., Arantes M., Casimiro M., Pais D. \& Pina J.A.E. 2005. Caracterização morfológica do polígono arterial de Willis em Canis familiaris. Revta Port. Ciênc. Vet. 100:163-167.

Casals J.B., Pieri N.C.G., Feitosa M.L.T., Ercolin A.C.M., Roballo K.C.S., Barreto R.S.N., Bressan F.F., Martins D.S., Miglino M.A. \& Ambrósio C.E. 2011. The use of animal models for stroke research: A review. Comp. Med. 61(4):305-313.

De Vrise B. 1905. Sur Ia significtion morfologique de artéres cerebrales. Arch. Biol. 21:357-457.

Fawcett E. \& Blanchford J.V. 1905-1906. The circle of Willis: an examination of 700 specimens. J. Anat. Physiol. 40:63-70.

Ferreira C.G. 1998. Estudo Anatômico das Artérias da Base do Encéfalo de Suínos (Sus scrofa domesticus Linnaeus, 1758). Tese de Mestrado, Faculdade de Medicina Veterinária e Zootecnia, USP, São Paulo. 98p.

Ferreira C.G. \& Prada I.L.S. 2005. 0 circuito arterial da base do encéfalo em suínos (Sus scrofa domesticus Linnaeus, 1758), formação e comportamento. Braz. J. Vet. Res. Anim. Sci. 42(1): 53-60.

Ferreira J.R. 1997. Estudo anatômico das artérias da base do encéfalo do macaco prego (Cebus apella Linnaeus,1766). Tese de Doutorado, Faculdade de Medicina Veterinária e Zootecnia, USP, São Paulo. 192p.

Ferreira J.R., Luz De Abreu N.H., Pires J.S. \& Ribeiro B.N. 2005. 0 sistema carótico do encéfalo de primata neotropical, Anatomia da artéria inter-hemisférica (Cebus apella Linnaeus, 1766). Ciênc. Anim. Bras. 6(3):203-212.

Ferreira C.G. 2002. Origem, trajeto, ramificação e distribiição da artéria cerebral rostral e suínos (Sus scrofa domesticus Linnaeus, 1758). Tese de Doutorado, Faculdade de Medicina Veterinária e Zootecnia, USP, São Paulo. 404p.

Ferreira J.R. \& Prada I.L.S. 2001. Nomenclatura proposta para denominar as artérias da base do encéfalo do macaco-prego (Cebus apella L., 1766). Acta Scientiarum 23(2):635-643.

Franciolli A.L.R., Costa G.M., Mançanares C.A.F., Martins D.S., Ambrósio C.E., Miglino M.A. \& Carvalho A.F. 2007. Morfologia dos órgãos genitais masculinos de quati (Nasua nasua Linnaeus 1766). Biotemas 20(1):27-36.

Gillilan L.A. 1969. The arterial and venous blood supplies to the cerebelum of primates. J. Neuropathol. Exp. Neurology 28:295-307.

Glidden E.M.E. \& Degaris C.R. 1936. Arteries of the chimpanzee. Am. J. Anat. 58:501-527.

Gompper M.E. 2004. Correlations of coati (Nasua narica) social structure with parasitism by ticks and chiggers. In: Sánchez-Cordero V. \& Medellín R.A. (Eds), Contribuciones Mastozoológicas em Homenaje a Bernardo Villa. Instituto de Biología e Instituto de Ecología UNAM, Cidade do México, México, p.527-534.

Guerin J., Gouaze A. \& Lazorthes G. 1976. Le polygone de Willis de l'enfant et les facteurs de son modelage. Neurochirurgie 22(3):217-226.

Kassel N.F. \& Langfitt T.W. 1965. Variations in the Circle of Willis in Macaca mulatta. Anatomical Record 162:257-264. 
Kramer S.P. 1912. On the function of the circles of Willis. J. Exp. Med. 15:348-355

Lima E.M.M. 2006. Estudo anatômico das artérias da base do encéfalo em gatos (Felis catus domesticus). Ars Vet. 22(1):1-7.

Lockhart R.D., Hamilton G.F. \& Fyfe F.W. 1983. Anatomia do Corpo Humano. $2^{\mathrm{a}}$ ed. Guanabara Koogan, Rio de Janeiro. 669p.

Machado A. 1988. Neuroanatomia Funcional. $2^{\mathrm{a}}$ ed. Atheneu, Rio de Janeiro. 363 pp.

McKenna M.C. 1966. Paleontology and the origin of the primates. Folia Primatol. 4(1):1-25.

Miraglia T. \& Teixeira A.M. 1960. Appunti sulla struttura e sulla vascolarizzazione dell'ipofise Del sagüi (Callithrix jacchus). Excerpta Médica 13:199-218.

Nanda B.S. 1975. Blood supply to the brain, p.1594-1651. In: Getty R. (Ed.), Sisson Grosman's Anatomy of the Domestic Aninals. W.B. Saunderes, Philadelphia.

Paiva-de-Souza A.V., Xavier-Silva B., Antunes M.S., Hernandez J.M.F., Scherer P.O. \& Abidu-Figueiredo M. 2010. Frequência da artéria caroticobasilar em eqüinos mestiços: estudo anatômico destinado a pesquisa experimental e ao diagnóstico por imagem. Pesq. Vet. Bras. 30(8):685-688.

Pieri N.C.G., Mançanares C.A.F., Bertassoli B., Lima J.M.N., Thomaz J.M. \& Carvalho A.F. 2011. Classificação morfofuncional dos dentes de quati, Nasua nasua. Pesq. Vet. Bras. 31(5):447-451.

Puchardes-Orts A., Nomblela-Gomes M. \& Ortuno-Pacheco G. 1975. Variation in form of circle of Willis: some anatomical and embryological considerations. Anatomical Record 185:119-124.

Reckziegel S.H., Lindemann T. \& Campos R.A. 2001. Systematic study of the brain base arteries in capybara (Hydrochoerus hydrochaeris). Braz. J. Morphol. Sci. 18(2):103-110.

Rohen J.W. \& Yokochi C. 1993. Anatomia Humana: Atlas fotográfico de anatomia sistêmica e regional. Ed. Manole, São Paulo. 450p.

Rouviere H. 1959. Anatomia Humana Descriptiva y Topografia. Bailly-Bailliere. Barcelona. 469p.

Russel J.K. 1996. Timing of reproduction by coatis (Nasua narica) in relation to fluctuations in food resources, p.413-431. In: Leigh Jr E.G., Rand A.S. \& Windsor D.M. (Eds), The Ecology of a Tropical Forest. $2^{\text {nd }}$ ed. Smithsonian Institution Press, Washington, DC.

Silva R.A. \& Ferreira J.R. 2002. Estudo das artérias cerebelares do macaco-prego. Considerações sobre a nomenclatura (Cebus apella L.1766). Braz. J. Vet. Res. Anim. Sci. 39(6):296-300

Shellshear L.L. 1927. The arteries of the brain of the Orang-Utan. J. Anat. 61:167-197.

Shellshear L.L. 1930. The arterial supply of the cerebral cortex in the chimpanzee (Anthropithecus troglodytes). J. Anat. 65:45-87.

Souza M.M.G., Teixeira A.M.C. \& Miraglia T. 1962. Dados sobre a vascularização do encéfalo do sagüi (Callinthrix jacchus). Folia Cl. Biol.31:104114.

Tandler J. 1898. Zur Vergleichenden Anatomie der Kopfarterien bei den Mammalia. Denkschrift der Akademie der Wissenschaften, Wien, 67: 677-784.

Testut L. 1911. Traité d'Anatomie Humaine. $6^{\text {iéme }}$ ed. Octave Doin, Paris. 944p.

WAVA 1992. Nomina Anatomica Veterinaria. $4^{\text {th }}$ ed. World Association of Veterinary Anatomists, Zürich. Cornell University, Ithaca. 166p. 